\section{A simple lyophilizer for direct line sublimation}

R. KEITH FARRELL AND S. D. JOHNSTON From the Endoparasite Vector Pioneering Research Laboratory, Veterinary Sciences Research Division, ARS, USDA, Washington State University, Pullman, WA

At a time of diminishing budgets in most laboratories simple home-made devices can sometimes function as efficiently as more expensive, commercially available ones. A lyophilization device modified for use in this laboratory is shown in the accompanying diagram. The dimensions are easily modified because of the basic simplicity of design.

The device consists of a vacuum desiccator, I, with glass lid removed. A doughnut-shaped piece of aluminium alloy, $\mathrm{D}$, is used to hold a commercial stainless steel beaker, A. The doughnut is turned on a lathe so that a gasket to engage the rim of the beaker is created. A lathe is used also to groove the opposite side for a large I-ring seal, G, to seal the doughnut-shaped aluminium cap against the rim of the vacuum jar. A hole is drilled through the doughnut-shaped piece of aluminium for the outlet port, C, which is constructed on the lathe to house again an O-ring seal, $E$, held against nut $F$. This ring is threaded so that pressure can be applied to the seal by the flange on the outlet port of the lyophilizer.

The stainless steel beaker forms a condenser to trap moisture. Refrigerants used can be either dry ice/alcohol or liquid nitrogen, and can be used with a cold finger to have automatic fill as desired. A plate, $\mathbf{H}$, is formed of another piece of doughnut-shaped metal, which makes a level surface if petri dishes, vials, or other containers need to be held level; this replaces the performated plate in the usual desiccator.

The vacuum pump is attached to the outlet port, $C$, and the material to be lyophilized is placed on the plate $H$. The large aluminium doughnut plus beaker is lowered into position, and vacuum is applied, creating a seal at O-rings B, E, and G. Vacuum gauges, inlets, dry nitrogen, etc, can be attached by Y-tubes distal to the outlet port. Lyophilization with the device is efficient in that there is a direct flow from the material to be dried to the condensing surface without the interference of small ports. The fact that a small drop of water can produce a large volume of water vapour is often overlooked

Received for publication 18 September 1972.

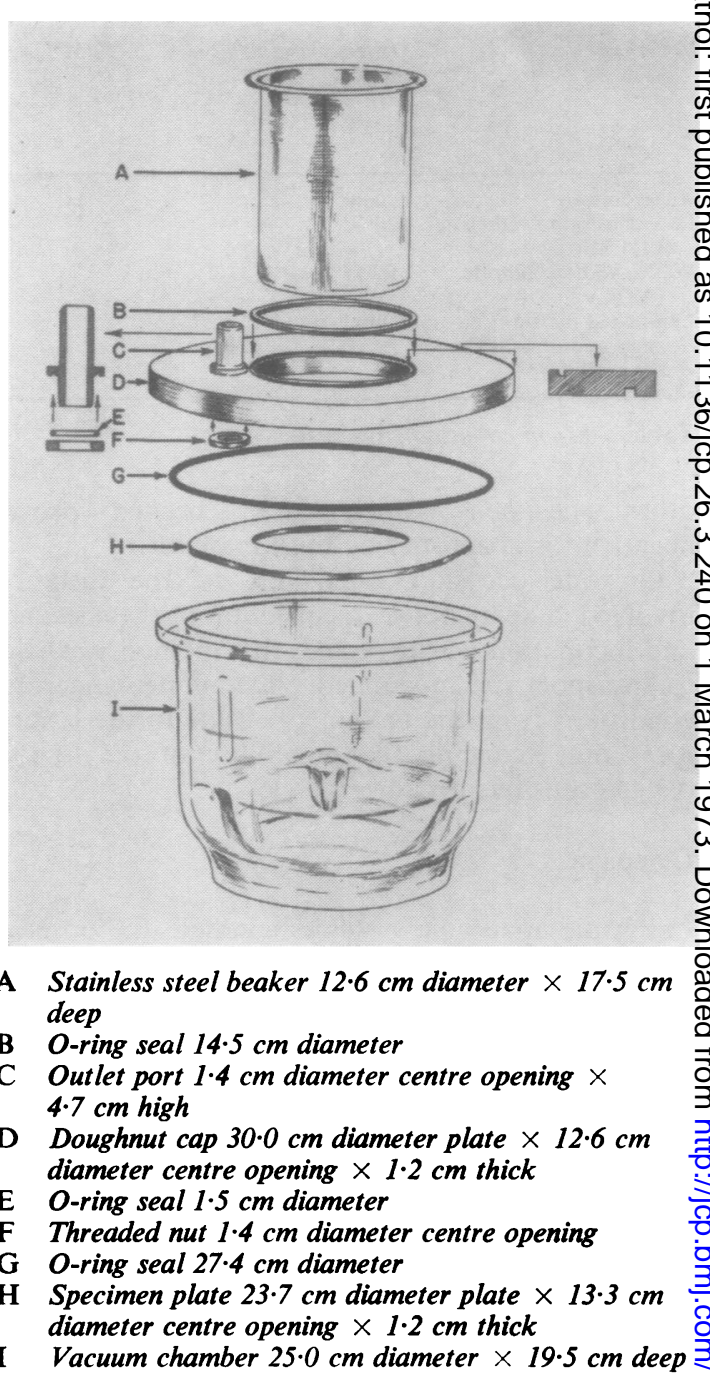

in the design of multiple port lyophilization devices (Harris, 1954).

Pancreatic enzymes frozen as thin films on suro faces of small glass beakers, virus-laden chorios allantoic membranes frozen flat in petri dishess్ ampoules frozen in Pyrex beakers, and rubber 0 stoppered ampoules containing viral and bacteria material have all been successfully lyophilized in the device.

We have routinely broken the vacuum with chilled super-dried nitrogen and removed the doughnut an $\Phi$ stainless steel beaker as one unit. Fluted stoppere $\vec{\phi}$ ampoules were then placed in a separate capper re-evacuated and sealed; ampoules to be flame-seale 8 were removed in the upright position and sealed. 